University of Wollongong

Research Online

Faculty of Informatics - Papers (Archive)

Faculty of Engineering and Information

Sciences

2007

\title{
Modulation solutions for nematicon propagation in non-local liquid crystals
}

Antonmaria Minzoni

Universidad Nacional Autónoma de México

Noel Smyth

University of Edinburgh

Annette L. Worthy

University of Wollongong, annie@uow.edu.au

Follow this and additional works at: https://ro.uow.edu.au/infopapers

Part of the Physical Sciences and Mathematics Commons

\section{Recommended Citation}

Minzoni, Antonmaria; Smyth, Noel; and Worthy, Annette L.: Modulation solutions for nematicon propagation in non-local liquid crystals 2007.

https://ro.uow.edu.au/infopapers/2653

Research Online is the open access institutional repository for the University of Wollongong. For further information contact the UOW Library: research-pubs@uow.edu.au 


\title{
Modulation solutions for nematicon propagation in non-local liquid crystals
}

\author{
Abstract \\ The propagation of solitary waves, so-called nematicons, in a nonlinear nematic liquid crystal is \\ considered in the nonlocal regime. Approximate modulation equations governing the evolution of input \\ beams into steady nematicons are derived by using suitable trial functions in a Lagrangian formulation of \\ the equations for a nematic liquid crystal. The variational equations are then extended to include the \\ effect of diffractive loss as the beam evolves. It is found that the nonlocal nature of the interaction \\ between the light and the nematic has a significant effect on the form of this diffractive radiation. \\ Furthermore, it is this shed radiation that allows the input beam to evolve to a steady nematicon. Finally, \\ excellent agreement is found between solutions of the modulation equations and numerical solutions of \\ the nematic liquid-crystal equations. \\ Disciplines \\ Physical Sciences and Mathematics \\ Publication Details \\ Minzoni, A., Smyth, N. F. \& Worthy, A. L. (2007). Modulation solutions for nematicon propagation in non- \\ local liquid crystals. Optical Society of America. Journal B: Optical Physics, 24 (7), 1549-1556.
}




\title{
Modulation solutions for nematicon propagation in nonlocal liquid crystals
}

\author{
Antonmaria A. Minzoni, ${ }^{1}$ Noel F. Smyth, ${ }^{2, *}$ and Annette L. Worthy ${ }^{3}$ \\ ${ }^{1}$ Fenomenos Nonlineales y Mecánica, Department of Mathematics and Mechanics, Instituto de Investigación en \\ Matemáticas Aplicadas y Sistemas, Universidad Nacional Autónoma de México, Apdo. 20-726, 01000 México, D.F. \\ ${ }^{2}$ School of Mathematics and Maxwell Institute for Mathematical Sciences, The King's Buildings, University of \\ Edinburgh, Edinburgh, Scotland, UK, EH9 3JZ \\ ${ }^{3}$ School of Mathematics and Applied Statistics, University of Wollongong, Northfields Avenue, Wollongong, New \\ South Wales, Australia, 2522 \\ *Corresponding author: N.Smyth@ed.ac.uk
}

Received December 21, 2006; revised February 26, 2007; accepted February 27, 2007; posted March 15, 2007 (Doc. ID 78332); published June 15, 2007

\begin{abstract}
The propagation of solitary waves, so-called nematicons, in a nonlinear nematic liquid crystal is considered in the nonlocal regime. Approximate modulation equations governing the evolution of input beams into steady nematicons are derived by using suitable trial functions in a Lagrangian formulation of the equations for a nematic liquid crystal. The variational equations are then extended to include the effect of diffractive loss as the beam evolves. It is found that the nonlocal nature of the interaction between the light and the nematic has a significant effect on the form of this diffractive radiation. Furthermore, it is this shed radiation that allows the input beam to evolve to a steady nematicon. Finally, excellent agreement is found between solutions of the modulation equations and numerical solutions of the nematic liquid-crystal equations. (C) 2007 Optical Society of America

OCIS codes: $190.5530,190.5940,160.3710,190.4400$.
\end{abstract}

\section{INTRODUCTION}

The possibility of guiding self-supporting, nonlinear beams (solitary waves) of light in the regime of large nonlocality in liquid crystals was shown in a remarkable series of experiments by Assanto et al. [1-4]. They named these light solitons, which resulted from the liquid crystal actively guiding the light, nematicons. Moreover, they also studied nematicon formation theoretically $[2,3]$. In this work the existence of static solitons was shown by using an expansion around the center of the beam, which gave a Gaussian beam and a parabolic profile for the optical axis [2,3]. This local expansion was matched to an exponentially decaying solution away from the center of the beam. This matching then gave a condition determining the relation between the nematicon's amplitude and the width of its waist. It also showed that the angle (director) profile is much wider than the waist of the beam. The dynamics of a nematicon was studied approximately by using the Fock-Leontovich equation in the limit of a large cell [3]. An expression for the evolution of the waist of the beam was then obtained, which showed its oscillation. It was further shown that steady solitons with no waist oscillation are recovered for specific values of the input power. The effect of diffractive loss by the soliton as it evolves was not considered.

On the more abstract side, it is known, since the nematicon equations (1) and (2) below are the same as those for a thermoelastic waveguide, that the soliton solution is stable [5]. However, no results for the dynamics of the evolution of a beam-type boundary condition can be obtained using the Lyapunov function discussed in Ref. [5].
The purpose of the present work is to fill the gap between these abstract and approximate results. To this end, the dynamics of nematicon evolution in a liquid crystal is studied using an extension of the method of Ref. [6] for optical pulse propagation, which is based on a form of modulation theory that includes the effect of the dispersive radiation shed by a pulse solitary wave during its evolution. This method was further developed to apply to soliton propagation in liquid crystals in the local regime $[7,8]$. In a similar work, but for one rather than for two space dimensions, as in Refs. [7] and [8], a variational method was employed to obtain approximate Gaussian profiles for steady one-dimensional nematicons [9]. Gaussian profiles were found to be a good approximation near the peak of a one-dimensional nematicon but decayed too fast in its tail. Lagrangian methods were also employed to find approximations to the steady twodimensional solitary wave solutions of a nonlocal nonlinear Schrödinger (NLS) equation with a Gaussian kernel in the nonlinear, nonlocal term [10]. However, such a Gaussian kernel is not applicable to liquid crystals.

An initial beam evolves into a nematicon by undergoing a breathing motion accompanied by the shedding of linear diffractive radiation. The modulation equations for the beam evolution are obtained by using a suitable trial function in an averaged Lagrangian for the nematicon equations. These modulation equations have a family of fixed points that are the steady nematicon solutions, with conservation of energy selecting the final steady state. It is shown how the modulation equations, coupled with loss due to shed diffractive radiation, explain and reproduce 
cell boundary

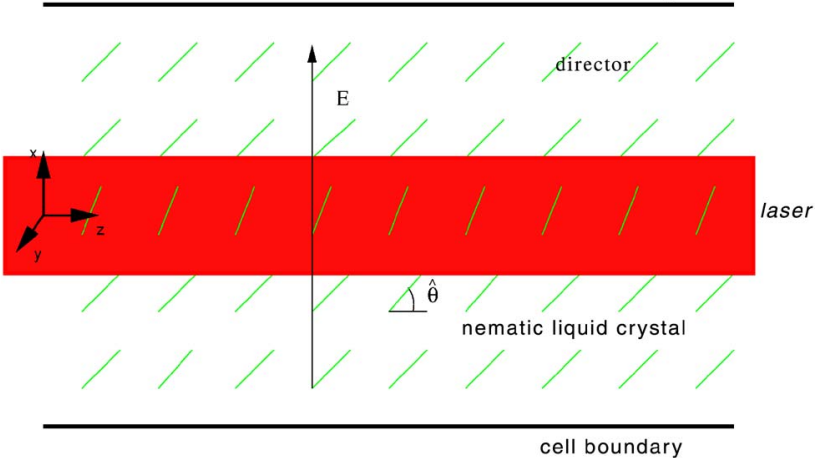

Fig. 1. (Color online) Schematic diagram of a liquid-crystal cell with a propagating polarized light beam.

quantitatively numerical solutions of the full equations governing the evolution of a nematicon.

\section{FORMULATION}

Let us consider a polarized beam propagating in the $z$ direction in a cell containing a nematic liquid crystal, such as was studied in works by Assanto et al. [1-3] and by García Reimbert et al. [7,8]. The geometry of the liquidcrystal cell is the same as that described in Ref. [7] and is shown in Fig. 1. The nematicon propagates in the $z$ direction, and the light is polarized such that its electric field is in the $x$ direction. The optical axis of the nematic is pretilted by a static electric field, also in the $x$ direction, with an angle $\hat{\theta}$ in order to overcome the Freédericksz threshold. The deviation from the pretilt angle due to the optical field is denoted by $\theta$. The slowly varying envelope of the optical electric field is denoted by $E$. The equations governing the propagation of a nematicon in the liquidcrystal cell are then [2,7]

$$
\begin{gathered}
i \frac{\partial E}{\partial z}+\frac{1}{2} \nabla^{2} E+\sin (2 \theta) E=0, \\
\nu \nabla^{2} \theta-q \sin (2 \theta)=-2|E|^{2} \cos (2 \theta) .
\end{gathered}
$$

Here $q$ is related to the external static electric field, and $\nu$ is the normalized elastic coefficient [8].

In the nonlocal limit it is known that the spatial extent of the response of the optical director is larger than the waist of the beam [2,3]. Moreover, it was also found in this same work that the deviation $\theta$ of the optical director from the equilibrium value is small. Under these nonlocal and small deviation assumptions, the nematicon equations (1) and (2) can be simplified to

$$
i \frac{\partial E}{\partial z}+\frac{1}{2} \nabla^{2} E+2 \theta E=0, \quad \nu \nabla^{2} \theta-2 q \theta=-2|E|^{2},
$$

which are the same equations as for the thermoelastic waveguide [5]. As the parameter $\nu$ increases, the response of the liquid crystal becomes more nonlocal, and the width of the optical axis becomes wider than the waist of the light beam. Note that in the local limit $\nu \rightarrow 0$, the system (3) can be reduced to the two-dimensional NLS equation, which predicts collapse of an input beam at a finite propa- gation distance $z$. The collapse of the beam in this local limit is stopped by the saturating nonlinearity of the nonlinear nematicon equations (1) and (2) [8]. In the present work, the dynamics of how nonlocality prevents collapse for the nonsaturable nematicon equations (3) will be studied.

\section{APPROXIMATE EQUATIONS}

To derive the approximate equations describing the evolution of a nematicon, the Lagrangian for the nematicon equations (3) is taken in polar coordinates:

$$
\begin{aligned}
L= & \int_{0}^{Z} \int_{0}^{\infty}\left[\operatorname{ir}\left(E^{*} E_{z}-E E_{z}^{*}\right)-r\left|E_{r}\right|^{2}+4 r \theta|E|^{2}-\nu r \theta_{r}^{2}\right. \\
& \left.-2 q r \theta^{2}\right] \mathrm{d} r \mathrm{~d} z,
\end{aligned}
$$

where the superscript * denotes the complex conjugate. This Lagrangian, which will be used to construct the approximate equations for nematicon evolution, is the same as that for thermoelastic waveguides [5].

As in Refs. [6-8], trial functions for the electric field $E$ and director angle $\theta$ are taken in the form

$$
E=a \operatorname{sech}(r / w) e^{i \sigma}+i g e^{i \sigma}, \quad \theta=\alpha \operatorname{sech}^{2}(r / \beta) .
$$

The electric field amplitude $a$, width $w$, phase $\sigma$, and the director angle amplitude $\alpha$ and width $\beta$ are functions of $z$. The parameter $g$ is also a function of $z$. The term $i g \exp (i \sigma)$ in the trial function for the electric field $E$ in Eqs. (5) represents the shed low-wavenumber diffractive radiation that sits under the evolving beam, forming a flat shelf or pedestal. This low-wavenumber, linear diffractive radiation has low group velocity and so cannot escape from the vicinity of the beam [6-8]. Perturbed inverse scattering for the NLS equation shows that for an optical pulse this low-wavenumber radiation is out of phase with the pulse, hence the factor $i$ in this radiation term [6]. As the shed radiation cannot remain flat away from the beam or pulse, it is assumed that $g$ is nonzero in a circular region of radius $l$ centered at the beam [6-8].

The electric field $E$ and director angle $\theta$ have halfwidths $w$ and $\beta$, respectively, in the trial functions Eqs. (5). In the strong nonlocal limit under consideration, it is expected that $\beta \gg w$, which leads to a more complicated system of modulation equations than for the local limit, for which both the electric field and the director angle have the same half-widths [8].

An averaged Lagrangian is now obtained by substituting the trial functions [Eqs. (5)] into the Lagrangian (4) and evaluating the $r$ integral. All the resulting integrals can be simply evaluated, except for the cross integral:

$$
\int_{0}^{\infty} 4 r \theta|E|^{2} \mathrm{~d} r=4 \alpha a^{2} \int_{0}^{\infty} r \operatorname{sech}^{2} \frac{r}{\beta} \operatorname{sech}^{2} \frac{r}{w} \mathrm{~d} r .
$$

This integral cannot be evaluated exactly unless $\beta=w$. To obtain useful, explicit approximate equations, the concept of equivalent functions is now used [8]. The function $\operatorname{sech}^{2}(r / \beta)$ is then replaced by the Gaussian $\exp \left(-r^{2} /(A \beta)^{2}\right)$, and $\operatorname{sech}^{2}(r / w)$ is replaced by the Gaussian $\exp \left(-r^{2} /(B w)^{2}\right)$, so that the integral (6) can now be explicitly evaluated. With this replacement, we have 


$$
\begin{aligned}
\int_{0}^{\infty} 4 r \theta|E|^{2} \mathrm{~d} r & =4 \alpha a^{2} \int_{0}^{\infty} r e^{-r^{2}\left(1 /(A \beta)^{2}+1 /(B w)^{2}\right)} \mathrm{d} r \\
& =\frac{2 \alpha a^{2} A^{2} B^{2} w^{2} \beta^{2}}{A^{2} \beta^{2}+B^{2} w^{2}}
\end{aligned}
$$

The new coefficients $A$ and $B$ are now determined by matching the Taylor series of the integral (6) with the hyperbolic secants to the result [Eq. (7)] using Gaussians to two terms in the limit $w \ll \beta$, which is the limit of interest in the nonlocal regime. This results in

$$
A=2 I_{2} / \sqrt{I_{x 32}}, \quad B=\sqrt{2 I_{2}},
$$

where

$$
\begin{gathered}
I_{2}=\int_{0}^{\infty} x \operatorname{sech}^{2} x \mathrm{~d} x=\ln 2, \\
I_{x 32}=\int_{0}^{\infty} x^{3} \operatorname{sech}^{2} x \mathrm{~d} x=1.352301002 \ldots
\end{gathered}
$$

The error in this Taylor-series matching is $O\left((w / \beta)^{3}\right)$. For the comparison example of Fig. 3, the steady-state values of $w$ and $\beta$ are $w_{0}=1.6768$ and $\beta_{0}=3.9647$, giving an error in matching of $O\left(8 \times 10^{-2}\right)$. In general, the larger $\nu$ is, the smaller $(w / \beta)^{3}$ is.

It might be thought that, since the hyperbolic secants of the trial functions have been replaced by equivalent Gaussians to evaluate the cross integral, it might have been better to use Gaussians for the trial functions [Eqs. (5)]. However, it was found that if this were done the resulting approximate equations would not give solutions in good agreement with numerical solutions, as was also the case for the nematicon equations in the local regime [8]. Furthermore, it was found that Gaussian initial conditions have a greater tendency to split into multiple solitons at moderate-to-high amplitudes than do hyperbolic secant initial conditions.

With the equivalent Gaussian approximation for the cross integral, the averaged Lagrangian is

$$
\begin{aligned}
\mathcal{L}= & -2\left(a^{2} w^{2} I_{2}+\Lambda g^{2}\right) \sigma^{\prime}-2 I_{1} a w^{2} g^{\prime}+2 I_{1} g w^{2} a^{\prime}+4 I_{1} a w g w^{\prime} \\
& -a^{2} I_{22}-4 \nu I_{42} \alpha^{2}-2 q I_{4} \alpha^{2} \beta^{2}+\frac{2 A^{2} B^{2} \alpha a^{2} \beta^{2} w^{2}}{A^{2} \beta^{2}+B^{2} w^{2}} .
\end{aligned}
$$

Here

$$
\Lambda=\frac{1}{2} l^{2}
$$

is the area of the shelf under the beam, modulo $2 \pi$. The integrals $I_{22}, I_{1}, I_{42}$, and $I_{4}$ are given by

$$
\begin{gathered}
I_{22}=\int_{0}^{\infty} x \operatorname{sech}^{2} x \tanh ^{2} x \mathrm{~d} x=\frac{1}{3} \ln 2+\frac{1}{6}, \\
I_{1}=\int_{0}^{\infty} x \operatorname{sech} x \mathrm{~d} x=2 C,
\end{gathered}
$$

$$
\begin{gathered}
I_{42}=\int_{0}^{\infty} x \operatorname{sech}^{4} x \tanh ^{2} x \mathrm{~d} x=\frac{2}{15} \ln 2+\frac{1}{60}, \\
I_{4}=\int_{0}^{\infty} x \operatorname{sech}^{4} x \mathrm{~d} x=\frac{2}{3} \ln 2-\frac{1}{6},
\end{gathered}
$$

where $C$ is the Catalan constant $C=0.915965594 \ldots$ [11].

Taking variations of the averaged Lagrangian (10) with respect to the parameters $a, w, \alpha, \beta, \sigma$, and $g$ results in the variational equations

$$
\frac{\mathrm{d}}{\mathrm{d} z}\left(I_{2} a^{2} w^{2}+\Lambda g^{2}\right)=0
$$

$$
\begin{gathered}
\frac{\mathrm{d}}{\mathrm{d} z}\left(I_{1} a w^{2}\right)=\Lambda g \frac{\mathrm{d} \sigma}{\mathrm{d} z} \\
I_{1} \frac{\mathrm{d} g}{\mathrm{~d} z}=\frac{I_{22} a}{2 w^{2}}-\frac{A^{2} B^{4} \alpha a w^{2} \beta^{2}}{\left(A^{2} \beta^{2}+B^{2} w^{2}\right)^{2}}, \\
I_{2} \frac{\mathrm{d} \sigma}{\mathrm{d} z}=-\frac{I_{22}}{w^{2}}+\frac{A^{2} B^{2} \alpha \beta^{2}\left(A^{2} \beta^{2}+2 B^{2} w^{2}\right)}{\left(A^{2} \beta^{2}+B^{2} w^{2}\right)^{2}}
\end{gathered}
$$

plus the algebraic equations

$$
\begin{gathered}
\alpha=\frac{A^{2} B^{2} \beta^{2} w^{2} a^{2}}{2\left(A^{2} \beta^{2}+B^{2} w^{2}\right)\left(2 \nu I_{42}+q I_{4} \beta^{2}\right)}, \\
\alpha=\frac{A^{2} B^{4} w^{4} a^{2}}{q I_{4}\left(A^{2} \beta^{2}+B^{2} w^{2}\right)^{2}} .
\end{gathered}
$$

These variational equations are the equations governing the evolution of the nematicon.

By Nöther's theorem, the nematicon equations (3) have an energy conservation equation that can be obtained from the Lagrangian (4) from invariances in $z$. The averaged form of this energy conservation equation is then

$$
\begin{aligned}
\frac{\mathrm{d} H}{\mathrm{~d} z} & =\frac{\mathrm{d}}{\mathrm{d} z} \int_{0}^{\infty} r\left[\left|E_{r}\right|^{2}-4 \theta|E|^{2}+\nu \theta_{r}^{2}+2 q \theta^{2}\right] \mathrm{d} r \\
& =\frac{\mathrm{d}}{\mathrm{d} z}\left(I_{22} a^{2}+4 \nu I_{42} \alpha^{2}+2 q I_{4} \alpha^{2} \beta^{2}-\frac{2 A^{2} B^{2} \alpha a^{2} w^{2} \beta^{2}}{A^{2} \beta^{2}+B^{2} w^{2}}\right)=0 .
\end{aligned}
$$

Since energy is conserved, this energy conservation equation can be used to determine the final steady nematicon state from the input beam.

When $\alpha$ is eliminated between the algebraic equations (17), the director angle width $\beta$ can be found as

$$
\beta^{2}=\frac{q I_{4} B^{2} w^{2}+\sqrt{q^{2} I_{4}^{2} B^{4} w^{4}+16 \nu q I_{42} I_{4} A^{2} B^{2} w^{2}}}{2 q A^{2} I_{4}} .
$$

With this expression for $\beta$, the amplitude $\alpha$ of the director is then given by the first of Eqs. (17). The amplitude and width of the optical axis are therefore given as algebraic functions of the nematicon in the electric field. 
At the fixed point of the approximate evolution equations (13)-(16), $g=0$. Hence Eq. (15) for $g$ gives that the steady amplitude $\hat{a}$ and width $\hat{w}$ of the nematicon are related by

$$
\hat{a}^{2}=\frac{I_{22}\left(A^{2} \hat{\beta}^{2}+B^{2} \hat{w}^{2}\right)^{3}\left(2 \nu I_{42}+q I_{4} \hat{\beta}^{2}\right)}{A^{4} B^{6} \hat{\beta}^{4} \hat{w}^{6}},
$$

which determines the family of steady nematicons with $\hat{a}$ as a function of $\hat{w}$. With this relation between $\hat{a}$ and $\hat{w}$, the energy equation (18) then determines the fixed point $\hat{w}$ from the parameters of the input beam. However, this energy equation is a transcendental equation in $\hat{w}$ and has to be solved numerically.

To complete the modulation equations, the radius $\ell$ of the shelf of low-wavenumber diffractive radiation under the evolving nematicon needs to be determined. As in previous work on NLS-type equations [6-8], this is achieved by linearizing the modulation equations (13)-(18) about the fixed points $\hat{a}, \hat{w}, \hat{\alpha}$, and $\hat{\beta}$. The fixed point of the modulation equations is then found to be a center whose frequency is related to $\Lambda$. As in Ref. [6], which was later confirmed by Ref. [12], the frequency of this center is then equated to the soliton frequency $\hat{\sigma}^{\prime}$ at the fixed point, which results in $\Lambda$, and hence $\ell$, in terms of the fixedpoint nematicon parameters. This determination of $\ell$, while straightforward in principle, is involved owing to the fact that five variables are involved in the linearization. The details are then given in Appendix A, with the final result for $\Lambda$ being

$$
\Lambda=-\frac{\hat{\sigma}^{\prime} I_{1}^{2}\left(\hat{w}^{2}+2 \hat{a} \hat{w} \Theta\right)}{Q},
$$

with $\Theta$ and $Q$ given in Appendix A.

The set of modulation equations governing the evolution of the nematicon is then Eqs. (14)-(16), the first of Eqs. (17) and Eqs. (18) and (19), with the shelf radius determined by Eq. (21). Note that the mass variational equation (13) has been replaced by the energy equation (18), which can be done, since the energy equation can be obtained by a suitable combination of the variational equations (13)-(16). As shown in Appendix A, these modulation equations predict that the nematicon will oscillate around the fixed point as it evolves. However, these modulation equations are not complete, as the effect of the linear diffractive radiation shed as the beam evolves has not been included. The determination of the effect of this shed radiation is the subject of Section 4 . When this radiation is included, the beam stabilizes to the steady state by undergoing oscillations in its waist width [3], in the manner of a damped simple harmonic oscillator.

\section{DIFFRACTIVE RADIATION LOSS}

To obtain good agreement with numerical solutions, the effect of the linear diffractive radiation shed by the beam as it evolves must be included [6-8]. The shed radiation has small amplitude relative to the beam and, thus being governed by the linearized electric field equation of (3), is

$$
i \frac{\partial E}{\partial z}+\frac{1}{2} \frac{\partial^{2} E}{\partial r^{2}}+\frac{1}{2 r} \frac{\partial E}{\partial r}=0 .
$$

This linearized equation was solved using Laplace transforms [7], so the details will not be repeated here. However, there is an important difference in the shelf of diffractive radiation under the $E$ beam, this being due to the nonlocal interaction between the electric field and the director. This difference can be seen in Fig. 2, which shows the full numerical solution of the nematicon equations (1) and (2). It can be seen that the shelf under the $E$ beam extends well beyond it and has the form of a truncated cone. This extended shelf is due to the nonlocal interaction with the director as the director beam has a long tail due to the large value of $\nu$. This in turn forces the electric field to have a long extension. The shelf of diffractive radiation sitting under the electric field beam therefore has two components. The inner portion of this shelf of radius $\ell$ was considered in Section 3 and is due to the resonant interaction of zero-wavenumber diffractive radiation with the beam. The outer portion of the shelf is due to the nonlocal forcing of the optical axis and has a radius $\rho$, which is related to the half-width $\beta_{1 / 2}$ $=\operatorname{sech}^{-1}(1 / \sqrt{2}) \beta$ of the optical disturbance of width $\beta$. This division of the shelf into two components, an inner shelf
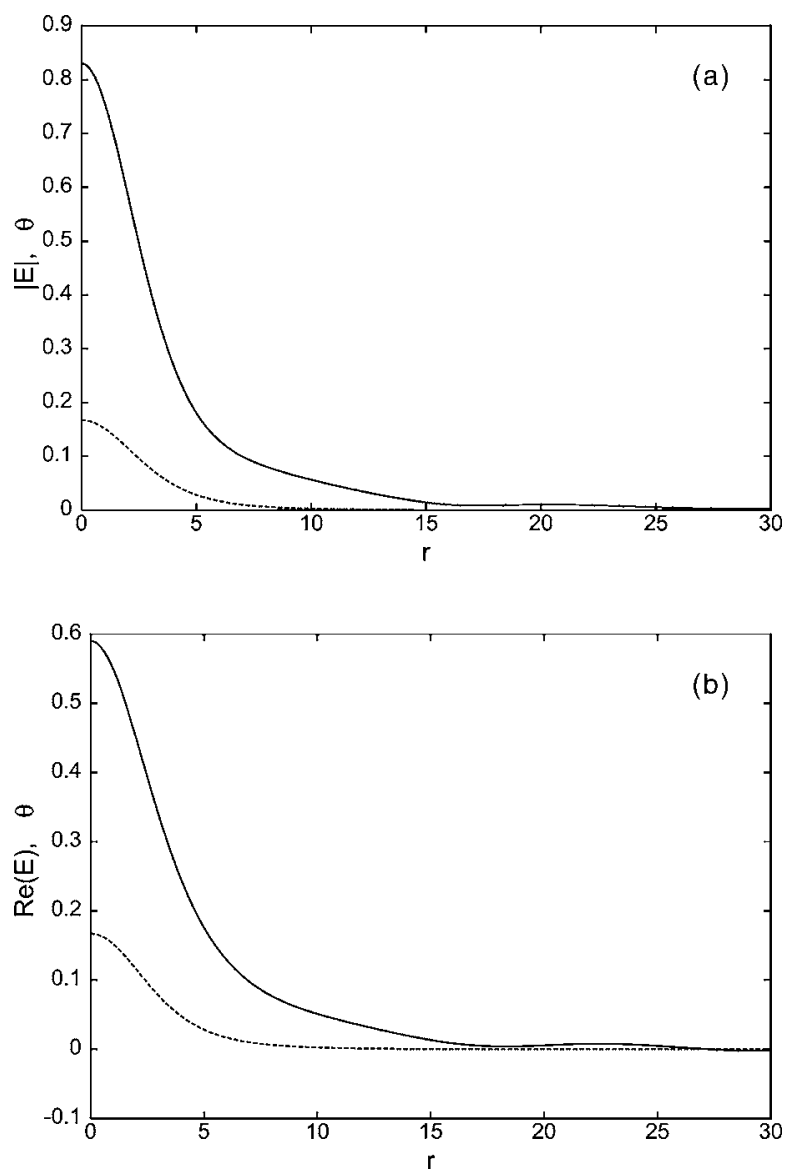

Fig. 2. Numerical solution nematicon equations (1) and (2) at $z=400$ for the initial values $a=0.5, w=4$, with $\alpha$ and $\beta$ determined by the first of Eqs. (17) and Eq. (19). The parameter values are $q=2$ and $\nu=10$. (a) Solution for $|E|$ : solid curve; solution for $\theta$ : dashed curve. (b) Solution for $\operatorname{Re}(E)$ : solid curve; solution for $\theta$ : dashed curve. 
and an outer shelf, was also found by Ref. [12] for perturbed vector solitons of the coupled NLS equations describing birefringent optical fibers. These two components of the shelf were shown to be due to two distinct types of eigenfunction of the continuous spectrum for the equations describing the perturbation.

The radius $\rho$ of the outer portion of the shelf under the electric field beam can be estimated from the numerical solution shown in Fig. 2 as approximately $\rho=15$. Solutions of the modulation equations give that $\beta$ $=2.18853 \ldots$ at $z=400$ for the initial conditions of Fig. 2 . Hence an estimation for the radius of the outer portion of the shelf is $\rho=7 \beta_{1 / 2}$, so that

$$
\widetilde{\Lambda}=\frac{1}{2} \rho^{2},
$$

in analogy with the relation between $\ell$ and $\Lambda$. Even though these values of $\rho$ and $\tilde{\Lambda}$ have been found for a specific initial condition, they will be found to give good agreement with numerical solutions for all initial conditions.

The diffractive radiation loss is now added to the modulation equations (14)-(16), the first of Eqs. (17), and Eqs. (18) and (19), which results in Eq. (15) being modified to $[7,8]$

$$
I_{1} \frac{\mathrm{d} g}{\mathrm{~d} z}=\frac{I_{22} a}{2 w^{2}}-\frac{A^{2} B^{4} \alpha a w^{2} \beta^{2}}{\left(A^{2} \beta^{2}+B^{2} w^{2}\right)^{2}}-2 \delta g
$$

where the loss coefficient $\delta$ is

$$
\delta=-\frac{\sqrt{2 \pi} I_{1}}{32 e R \tilde{\Lambda}} \int_{0}^{z} \frac{\pi R\left(z^{\prime}\right) \ln \left(\left(z-z^{\prime}\right) / \widetilde{\Lambda}\right)}{\left\{\left[\frac{1}{4} \ln \left(\left(z-z^{\prime}\right) / \widetilde{\Lambda}\right)\right]^{2}+3 \pi^{2} / 16\right\}^{2}+\pi^{2}\left[\ln \left(\left(z-z^{\prime}\right) / \widetilde{\Lambda}\right)\right]^{2} / 16} \frac{\mathrm{d} z^{\prime}}{\left(z-z^{\prime}\right)}
$$

$$
R^{2}=\frac{1}{\widetilde{\Lambda}}\left[I_{2} a^{2} w^{2}-I_{2} \hat{a}^{2} \hat{w}^{2}+\widetilde{\Lambda} g^{2}\right]
$$

For the local regime considered by Refs. [7] and [8], $\tilde{\Lambda}$ $=\Lambda$ as the optical axis and the electric field have the same width, and the shelf under the electric field beam is the usual NLS shelf [6]. The loss coefficient [Eq. (25)] differs from that of García Reimbert et al. [7,8] by the replacement of $\Lambda$ by $\tilde{\Lambda}$ in the loss coefficient $\delta$ and $R$. This is because $\Lambda$ in these terms comes from the total radius of the shelf under the beam [7].

The final modulation equations describing the evolution of the nematicon are then Eqs. (14) and (16), the first of Eqs. (17), (18)-(19), and (24). The solution of these modulation equations will be compared with full numerical solutions of the nematicon equations (1) and (2) in Section 5 .

\section{RESULTS}

In this section, full numerical solutions of the nematicon equations (1) and (2) will be compared with solutions of the approximate equations (14) and (16), the first of Eqs. (17), (18)-(19), and (24). The numerical method used to solve the nematicon equations (1) and (2) is the same as that of Ref. [8], so only a brief description of the method will be given here. The electric field equation (1) was solved using a pseudospectral method [13]. The main difference with the method described in Ref. [13] is that the stepping in the $z$ direction is performed in Fourier space using a fourth-order Runge-Kutta method rather than in physical space using a leap-frog scheme. The $r$ derivatives are calculated using fast Fourier transforms. To numerically solve the optical axis equation (2), it was rewritten in the form

$$
\nu \frac{\partial^{2} \theta}{\partial r^{2}}+\frac{\nu}{r} \frac{\partial \theta}{\partial r}-2 q \theta=q \sin (2 \theta)-2 q \theta-2|E|^{2} \cos (2 \theta) .
$$

The derivatives were calculated using standard secondorder finite differences, which gave a tridiagonal system. Together with the boundary conditions $\theta_{r}=0$ at $r=0$ and $\theta \rightarrow 0$ as $r \rightarrow \infty$, the resulting two-point boundary-value problem was solved using a Picard iteration. The modulation equations (14), (16), (18), and (24) were solved numerically using the standard fourth-order Runge-Kutta scheme.

The first comparison between the numerical solution and the solution of the approximate equations is shown in Fig. 3, for which the initial conditions are that $a=0.5$ and $w=4$ at $z=0$ with $q=2$ and $\nu=10$. It can be seen that there is excellent agreement in both the mean and the envelope of the amplitude oscillation, with the approximate mean, being the amplitude of the final steady nematicon, being slightly higher than the numerical mean. The agreement between the period of the solution of the modulation equations and that of the full numerical solution is excellent. This agreement is remarkable, given that the amplitude of the beam doubles over the initial amplitude during the oscillations and that the frequency of the modulation equations' solution was obtained by linearizing about the fixed point and then assuming that this result holds away from the fixed point. In the derivation of the modulation equations, it was assumed that for large $\nu$ the director response $\theta$ is small. While the electric field amplitude $a$ has a large amplitude oscillation, numerical solutions of the full nematicon equations (1) and (2) show that the director angle has a small oscillation amplitude of $\alpha=O(0.2)$. The reason that the electric field response is so large is that two-dimensional solitary waves for nonlocal NLS- 


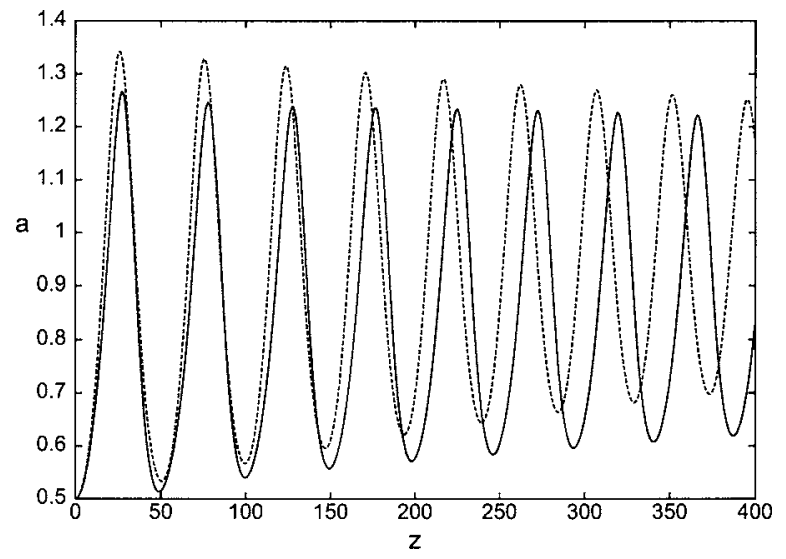

Fig. 3. Amplitude $a$ for the electric field $E$ as a function of $z$ for the initial conditions $a=0.5, w=4$, with $\alpha$ and $\beta$ determined by the first of Eqs. (17) and Eq. (19). The parameter values are $q$ $=2$ and $\nu=10$. Numerical solution of nematicon equations (1) and (2): solid curve; solution of modulation equations (14) and (16), the first of Eqs. (17), and Eqs. (18), (19), and (24): dashed curve.

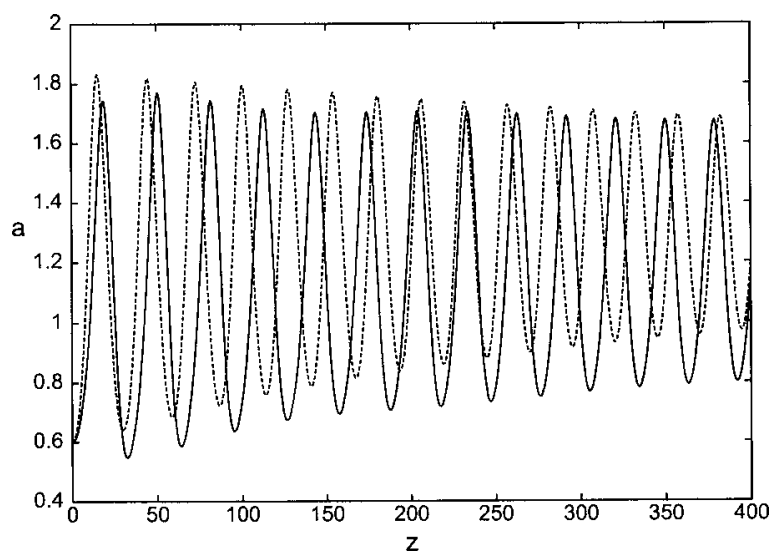

Fig. 4. Amplitude $a$ for the electric field $E$ as a function of $z$ for the initial conditions $a=0.6, w=4$, with $\alpha$ and $\beta$ determined by the first of Eqs. (17) and Eq. (19). The parameter values are $q$ $=2$ and $\nu=10$. Numerical solution of nematicon equations (1) and (2): solid curve; solution of modulation equations (14) and (16), the first of Eqs. (17), and Eqs. (18), (19), and (24): dashed curve.

type equations are close to instability. The amplitude grows to quite large values, becoming unstable, before nonlocality stops the growth [14].

The nematicon equations (1) and (2) have been nondimensionalized by the Rayleigh distance, so that the nematicon evolution of Figs. 3-6 is shown over much longer distances than are experimentally possible. This was done so that the figures show a number of oscillations and a significant degree of evolution to the steady state. Another point is that loss cannot be ignored over such long evolution distances $[15,16]$. Now that the basic approximate equations for nematicon evolution have been determined, the addition of dissipative terms could form the basis for further work.

Figure 4 shows a similar amplitude comparison for the initial conditions $a=0.6$ and $w=4$ with $q=2$ and $\nu=10$. The envelope and mean of the approximate and numerical solutions are again in good agreement, with, again, the mean of the approximate solution (i.e., the final steady state) being slightly higher. For this initial condi- tion, the period of the approximate solution is slightly shorter than the numerical period. In Section 5 the radiative loss was fixed by determining the extended length of the shelf under the electric field beam from a specific numerical example, which was for the initial condition of Fig. 3. It can be seen that the decay rate of the oscillations of the solution of the approximate equations is still in very good agreement with the numerical decay rate for this same value of $\tilde{\Lambda}$.

Figure 5 shows the same amplitude comparison as for the two previous figures for the initial values $a=0.7$ and $w=4$ with $q=2$ and $\nu=10$. Again, the mean of the approximate oscillations is slightly higher than the numerical mean, with the decay rate in good agreement with the numerical value. However, the period of the oscillations of the modulation solution is somewhat shorter than the numerical period, with an additional phase difference. As the amplitude of the beam nearly quadruples during the oscillation, this is remarkable agreement, given that the approximate period was determined by linearization about the fixed point. This example is further justification for choosing the value of $\widetilde{\Lambda}$ from a specific example. As the modulation equations form a nonlinear oscillator, the difference in the amplitudes as given by the numerical and approximate solutions is linked to this difference in the period. These comments also explain the amplitude and period differences between the numerical and approximate solutions of Fig. 4.

The final amplitude comparison in Fig. 6 is for the lower initial amplitude $a=0.4$ with the same initial width $w=4$ and $q=2, \nu=10$. In contrast to the three previous examples, there is not good agreement between the numerical and the approximate amplitudes, while there is still good agreement in the period. The reason for the amplitude disagreement is that this initial condition is close to the threshold amplitude at $a=0.32$ for $w=4, q=2$, and $\nu$ $=10$, below which the input beam decays into diffractive radiation and does not form a nematicon, in which case good agreement is not expected. The oscillation period agreement is better than in Figs. 4 and 5 as the amplitude

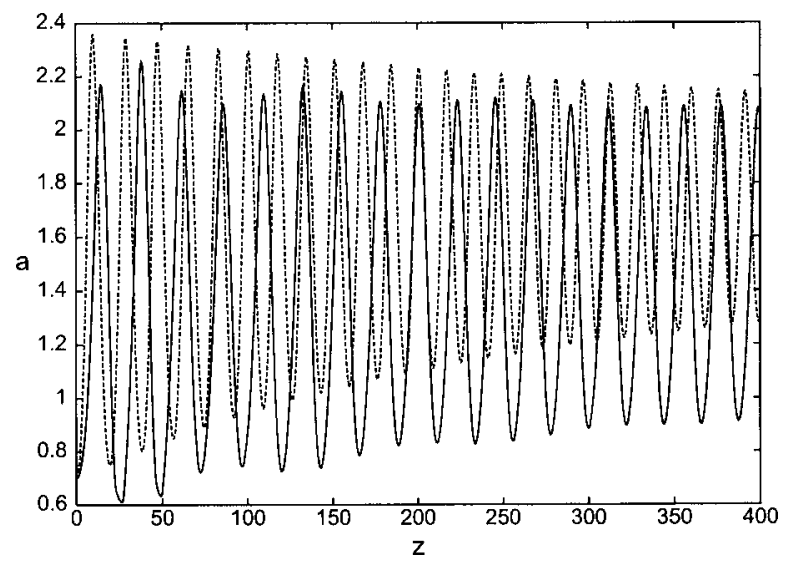

Fig. 5. Amplitude $a$ for the electric field $E$ as a function of $z$ for the initial conditions $a=0.7, w=4$, with $\alpha$ and $\beta$ determined by the first of Eqs. (17) and Eq. (19). The parameter values are $q$ $=2$ and $\nu=10$. Numerical solution of nematicon equations (1) and (2): solid curve; solution of modulation equations (14) and (16), the first of Eqs. (17), and Eqs. (18), (19), and (24): dashed curve. 


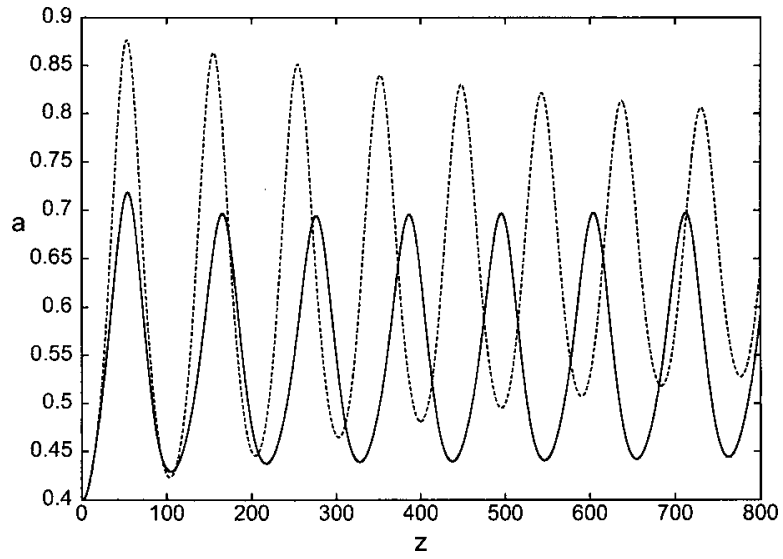

Fig. 6. Amplitude $a$ for the electric field $E$ as a function of $z$ for the initial conditions $a=0.4, w=4$, with $\alpha$ and $\beta$ determined by the first of Eqs. (17) and Eq. (19). The parameter values are $q$ $=2$ and $\nu=10$. Numerical solution of nematicon equations $(1)$ and (2): solid curve; solution of modulation equations (14) and (16), the first of Eqs. (17), and Eqs. (18), (19), and (24): dashed curve.

in this case only doubles during the oscillations, rather than triples as in these previous figures. The modulation equations are then closer to their linearized form about the fixed point, from which the shelf radius $\ell$, which determines the oscillation period of the modulation equations, was determined.

\section{CONCLUSIONS}

The evolution of beamlike initial conditions into steady nematicons has been studied for the regime of strongly nonlocal nematic liquid crystals. Modulation equations for the evolution of the beam were derived by using suitable trial functions in a Lagrangian formulation of the nematicon equations. When terms that include the effect of diffractive loss as the beam evolves were added to these modulation equations, excellent agreement was found between numerical solutions and solutions of the modulation equations. In the strongly nonlocal regime studied in the present work, the width of the beam in the optical axis is much larger than that of the electric field. This larger optical axis width has the effect of increasing the length of the shelf of linear diffractive radiation under the electric field beam over that studied in previous works [6-8]. This, in turn, leads to increased diffractive loss from the nematicon as it evolves.

The approach of the present work completes that of Conti et al. [3], since the effect of the diffractive radiation shed as the beam evolves is taken into account. It has been shown that the shed radiation acts as a damping that settles the initial condition to the steady nematicon. The evolving beam undergoes a breathing motion in its waist, in the manner of a damped simple harmonic oscillator.

As final comments, some remarks are needed on the nature of the approximations made to derive the evolution equations for the nematicon. The trial functions used for the beam electric field and the director angle include the effect of nonlocality, introducing an additional variational parameter. To evaluate a coupling integral between the electric field and the director angle, equivalent Gaus- sians were used to replace the hyperbolic secant trial functions in this integral. Using this replacement, an explicit averaged Lagrangian could be obtained. Finally, as remarked above, the nonlocality introduced an additional feature in the shelf of diffractive radiation under the evolving nematicon. The analysis undertaken shows how the results are independent of the particular nematicon system of the present work. The present analysis could then be useful in studying other soliton problems that involve some type of nonlocal behavior.

\section{APPENDIX A: SHELF RADIUS}

The radius $\ell$ of the shelf of diffractive radiation under the evolving nematicon is determined by linearizing the modulation equations (13)-(18) about the fixed point with

$$
\begin{aligned}
& a=\hat{a}+a_{1}, \quad w=\hat{w}+w_{1}, \quad \alpha=\hat{\alpha}+\alpha_{1}, \\
& \beta=\hat{\beta}+\beta_{1}, \quad g=g_{1}, \quad \sigma=\hat{\sigma}+\sigma_{1},
\end{aligned}
$$

where $\left|a_{1}\right| \ll \hat{a},\left|w_{1}\right| \ll \hat{w},\left|\alpha_{1}\right| \ll \hat{\alpha},\left|\beta_{1}\right| \ll \hat{\beta},\left|g_{1}\right| \ll 1$, and $\left|\sigma_{1}\right|$ $\ll \hat{\sigma}$. In a straightforward, but tedious, calculation, it is found on eliminating all the derivatives in terms of $a_{1}^{\prime}$ and then eliminating $a_{1}^{\prime}$ in terms of $g_{1}$ that

$$
\frac{\mathrm{d}^{2} g_{1}}{\mathrm{~d} z^{2}}-\frac{Q \Lambda \hat{\sigma}^{\prime}}{I_{1}^{2}\left(\hat{w}^{2}+2 \hat{a} \hat{w} \Theta\right)} g_{1}=0,
$$

where

$$
\begin{aligned}
\Theta= & \frac{2 I_{22} D^{2} \hat{a}-A^{2} B^{2} D \hat{\beta}^{2} \hat{w}^{2} \hat{a}\left(2 \hat{\alpha}+\Gamma_{1} \hat{a}\right)}{A^{2} B^{2} \hat{\beta} \hat{w} \hat{a}^{2}\left(2 A^{2} \hat{\alpha} \hat{\beta}^{3}+\Gamma_{2} D \hat{\beta} \hat{w}+2 B^{2} \Gamma_{3} \hat{\alpha} \hat{w}^{3}\right)}, \\
Q= & Q_{1}-\frac{I_{22}}{2 A^{2} B^{4} \hat{\alpha} \hat{\beta}^{2} \hat{w}^{4}}\left[Q_{2}+\Theta Q_{3}\right], \\
Q_{1}= & \frac{I_{22}}{2 \hat{w}^{2}}\left(1-\frac{2 \hat{a}}{\hat{w}} \Theta\right), \quad Q_{2}=\hat{\alpha}^{2} \hat{\beta}^{2} \hat{w}^{2}+\Gamma_{1} \hat{\beta}^{2} \hat{w}^{2} \hat{a}, \\
Q_{3}= & 2 \hat{\alpha} \hat{\beta}^{2} \hat{w} \hat{a}+\Gamma_{2} \hat{\beta}^{2} \hat{w}^{2} \hat{a}+2 \Gamma_{3} \hat{\alpha} \hat{\beta} \hat{w}^{2} \hat{a} \\
& -4 D^{-1} \hat{\alpha} \hat{\beta}^{2} \hat{w}^{2} \hat{a}\left(A^{2} \Gamma_{3} \hat{\beta}+B^{2} \hat{w}\right), \\
\Gamma_{1}= & \frac{2 A^{2} B^{4} \hat{w}^{4} \hat{a}}{q I_{4} D^{2}}, \quad \Gamma_{2}=\frac{4 A^{4} B^{4} \hat{\beta}^{3} \hat{w}^{2}\left(\hat{\beta}-\Gamma_{3} \hat{w}\right)}{q I_{4} D^{3}}, \\
\Gamma_{3}= & \frac{B^{2} \hat{w}\left(q I_{4} \hat{\beta}^{2}+4 \nu I_{3}\right)}{q I_{4} \hat{\beta}\left(2 A^{2} \hat{\beta}^{2}-B^{2} \hat{w}^{2}\right)}, \quad D=A^{2} \hat{\beta}^{2}+B^{2} \hat{w}^{2} .
\end{aligned}
$$

As in Ref. [6], the frequency of the simple harmonic motion equation (A2) is now matched to the steady nematicon oscillation frequency $\hat{\sigma}^{\prime}$, given by Eq. (16) with $a, w$, $\beta$, and $\alpha$ replaced by their fixed-point values. This results in the radius $\ell$ of the shelf of low-wavenumber diffractive radiation under the beam being determined by

$$
\Lambda=-\frac{\hat{\sigma}^{\prime} I_{1}^{2}\left(\hat{w}^{2}+2 \hat{a} \hat{w} \Theta\right)}{Q} .
$$


The fact that the shelf under the soliton oscillates at the frequency of the soliton was also shown by Yang [12] for the vector soliton solutions of the coupled NLS equations for a birefringent fiber by examining the eigenfunctions for the equations governing a small perturbation from the soliton state.

This research was supported by the Engineering and Physical Sciences Research Council under grant EP/ C548612/1.

\section{REFERENCES}

1. G. Assanto, M. Peccianti, and C. Conti, "Nemations: optical spatial solitons in nematic liquid crystals," Opt. Photonics News Feb. 2003, pp. 44-48.

2. C. Conti, M. Peccianti, and G. Assanto, "Route to nonlocality and observation of accessible solitons," Phys. Rev. Lett. 91, 073901 (2003).

3. C. Conti, M. Peccianti, and G. Assanto, "Observation of optical spatial solitons in a highly nonlocal medium," Phys. Rev. Lett. 92, 113902 (2004).

4. M. Peccianti, C. Conti, G. Assanto, A. de Luca, and C. Umeton, "Routing of anisotropic spatial solitons and modulational instability in liquid crystals," Nature 432, 733-737 (2004).

5. E. A. Kuznetsov and A. M. Rubenchik, "Soliton stabilization in plasmas and hydrodynamics," Phys. Rep. 142, 103-165 (1986).

6. W. L. Kath and N. F. Smyth, "Soliton evolution and radiation loss for the nonlinear Schrödinger equation," Phys. Rev. E 51, 1484-1492 (1995).
7. C. García Reimbert, A. A. Minzoni, and N. F. Smyth, "Spatial soliton evolution in nematic liquid crystals in the nonlinear local regime," J. Opt. Soc. Am. B 23, 294-301 (2006).

8. C. García Reimbert, A. A. Minzoni, N. F. Smyth, and A. L. Worthy, "Large-amplitude nematicon propagation in a liquid crystal with local response," J. Opt. Soc. Am. B 23, 2551-2558 (2006).

9. P. D. Rasmussen, O. Bang, and W. Królikowski, "Theory of nonlocal soliton interaction in nematic liquid crystals," Phys. Rev. E 72, 066611 (2005).

10. A. I. Yakimenko, V. M. Lashkin, and O. O. Prikhodko, "Dynamics of two-dimensional coherent structures in nonlocal nonlinear media," Phys. Rev. E 73, 066605 (2006).

11. M. Abramowitz and I. A. Stegun, Handbook of Mathematical Functions with Formulas, Graphs, and Mathematical Tables (Dover, 1972).

12. J. Yang, "Vector solitons and their internal oscillations in birefringent nonlinear optical fibers," Stud. Appl. Math. 98, 61-97 (1997).

13. B. Fornberg and G. B. Whitham, "A numerical and theoretical study of certain nonlinear wave phenomena," Philos. Trans. R. Soc. London, Ser. A 289, 373-403 (1978).

14. W. Królikowski, O. Bang, N. I. Nikolov, D. Neshev, J. Whyller, J. J. Rasmussen, and D. Edmundson, "Modulation instability, solitons and beam propagation in spatially nonlocal nonlinear media," J. Opt. B: Quantum Semiclassical Opt. 6, S288-S294 (2004).

15. Y. Huang, Q. Guo, and J. Cao, "Optical beams in lossy non-local Kerr media," Opt. Commun. 261, 175-180 (2006).

16. M. Peccianti, A. De Rossi, G. Assanto, A. De Luca, C. Umeton, and I. C. Khoo, "Electrically assisted selfconfinement and waveguiding in planar nematic liquid crystal cells," Appl. Phys. Lett. 77, 7-9 (2000). 